\title{
Quetiapine, Olanzapine and Haloperidol Affect Human Plasma Lipid Peroxidation in vitro
}

\author{
Anna Dietrich-Muszalska ${ }^{\mathrm{a}}$ Bogdan Kontek $^{\mathrm{b}}$ Jolanta Rabe-Jabłońska ${ }^{\mathrm{a}}$ \\ a Department of Affective and Psychotic Disorders, Medical University of Lodz, and \\ ${ }^{b}$ Department of General Biochemistry, University of Lodz, Lodz, Poland
}

\section{Key Words}

Oxidative stress - Lipid peroxidation - Antipsychotics .

Quetiapine $\cdot$ Olanzapine $\cdot$ Haloperidol

\begin{abstract}
Objective: Oxidative injury in schizophrenia may be caused not only by pathophysiological processes but partly also by treatment with antipsychotics. The purpose of the present study was to examine and to compare the effects of quetiapine (QUE), olanzapine (OLA) and haloperidol (HAL), at final concentrations corresponding to doses used for treatment of acute episodes of schizophrenia, on plasma lipid peroxidation in vitro, measured by the level of thiobarbituric acid-reactive substances (TBARS). Methods: Blood from 30 healthy volunteers was collected into ACD (citric acid/citrate/ dextrose) solution. The drugs in form of active substances were dissolved in $0.01 \%$ dimethyl sulfoxide, added to plasma at the final concentrations [QUE (175 and $275 \mathrm{ng} / \mathrm{ml}$ ), OLA (20 and $40 \mathrm{ng} / \mathrm{ml}$ ), HAL ( 4 and $20 \mathrm{ng} / \mathrm{ml}$ )] and incubated for 1 and $24 \mathrm{~h}$ at $37^{\circ} \mathrm{C}$. The level of TBARS was measured spectrophotometrically (according to the Rice-Evans method, 1991). $R$ esults: The comparative study in vitro showed that QUE causes a decrease in the TBARS level in plasma, whereas HAL increases the plasma TBARS level. After $24 \mathrm{~h}$ of incubation of plasma with QUE or HAL (at lower and higher concentrations), the differences in TBARS levels between the drugs were significant $\left(p=5.9 \times 10^{-4}, p=2.2 \times 10^{-5}\right.$, respectively).
\end{abstract}

Conclusion: QUE and OLA, contrary to the prooxidative action of HAL, did not induce oxidative stress; moreover, QUE has antioxidant properties.

Copyright $\odot 2011$ S. Karger AG, Basel

\section{Introduction}

Antipsychotics are extensively used in the treatment of schizophrenia and other psychiatric disorders, psychotic mainly. Oxidative stress may contribute to specific aspects of schizophrenic symptomatology and complications of its treatment such as prominent negative symptoms, tardive dyskinesia (TD), and parkinsonian symptoms. The first-generation antipsychotics such as haloperidol (HAL) often cause extrapyramidal side effects: motor disturbances of TD and parkinsonism [1]. TD occurs in $20-40 \%$ of the patients, who are treated chronically with antipsychotics $[2,3]$. In spite of its high incidence, the pathophysiology of TD remains elusive. Chronic treatment with antipsychotics probably increases free radical production and oxidative stress [4]. A role that increased reactive oxygen species (ROS) and oxidative stress in the etiopathology of antipsychotics induced TD has been proposed $[5,6]$. Chronic use of antipsychotics is also reported to cause a decrease in the activity of antioxidant enzymes, superoxide dismutase and catalase [7].

\section{KARGER}

Fax +41613061234

E-Mail karger@karger.ch

www.karger.com
(C) 2011 S. Karger AG, Basel

0302-282X/11/0634-0197\$38.00/0

Accessible online at:

www.karger.com/nps
Anna Dietrich-Muszalska, MD, PhD

Department of Affective and Psychotic Disorders

Medical University of Lodz, Czechoslowacka 8/10

PL-92-216 Lodz (Poland)

Tel. +48 42691881 787, Fax +48 42675 7403, E-Mail tzn_lodz@ @ost.pl 
The second-generation antipsychotics (SGAs) may also have some effects on oxidative stress, measured by the level of lipid peroxidation in plasma. The effects of olanzapine (OLA) and quetiapine (QUE) on this process are explained only partially. QUE and OLA belong to the chemical class of benzisoxazole derivatives and have been found to be effective in the treatment of schizophrenic disorders, with therapeutic activity mediated through a combination of D2 and 5HT2 receptor antagonism [8]. Moreover, it has been shown that they have a very low potential for causing extrapyramidal symptoms (EPS) across the full dose range $[9,10]$. QUE and OLA may improve cognitive functions (attentional, motor, and visuomotor skills) as well as executive functions connected to the psychopathology of schizophrenia [10]. It seems that the antioxidant mechanism of QUE and OLA may contribute to the low incidence of EPS and movement disorders caused by these drugs in patients with schizophrenia, and may be effective in the treatment of TD caused by other antipsychotics $[10,11]$.

The aim of the study was to evaluate the effects of SGAs and establish whether there is a difference among OLA, QUE and HAL action regarding their influence on lipid peroxidation in human plasma, measured by the level of thiobarbituric acid-reactive substances (TBARS).

\section{Materials and Methods}

\section{Inclusion Criteria of Healthy Subjects}

Blood samples were taken from 30 healthy males aged between 25 and 29 years (average: 28.3, SD $=1.5$ years), without psychiatric, neurological or somatic disorders or a history of head injuries, lipid or carbohydrate metabolism disorders, with a normal body mass index, and not being treated with any drugs. Healthy subjects (no smokers) did not use any addictive substances and antioxidant supplementation, and their diet was balanced (meat and vegetables). They lived in similar socioeconomic conditions. Psychiatric examination (using the M.I.N.I. - Mini International Neuropsychiatric Interview [12]), and neurological and somatic examinations were performed.

All subjects signed a consent to the participation in the study, according to the protocol accepted by the Committee for Research on Human Subjects of the Medical University of Lodz (No. RNN/899/2000).

\section{Isolation of Plasma}

Human blood $(3 \times 7.5 \mathrm{ml})$ was collected into ACD solution (citric acid/citrate/dextrose; 5:1 v/v) between 8.00 and 8.30 a.m. and centrifuged for $20 \mathrm{~min}$ at $2,500 \mathrm{rpm}$ and $20^{\circ} \mathrm{C}$ in a Sigma $3 \mathrm{~K} 30$ centrifuge to obtain plasma. The drugs obtained from the manufacturers (QUE: Celon Pharma, Poland; OLA: Adamed, Poland; HAL: Polfa-Warsaw, Poland) in the form of active ingredients were dissolved in $0.01 \%$ dimethyl sulfoxide (Sigma). Drug solutions were added to $0.5 \mathrm{ml}$ of plasma (QUE at the final concentrations of 175 and $275 \mathrm{ng} / \mathrm{ml}$; OLA 20 and $40 \mathrm{ng} / \mathrm{ml}$; HAL 4 and $20 \mathrm{ng} / \mathrm{ml}$ ) and incubated for 1 and $24 \mathrm{~h}$ at $37^{\circ} \mathrm{C}$. The controls were plasma samples containing $0.01 \%$ dimethyl sulfoxide without drug. Metabolites of QUE and OLA were not investigated.

\section{Evaluation of Lipid Peroxidation Level}

In control samples and samples of plasma after the incubation with the drug, the concentrations of TBARS were measured spectrophotometrically, according to the Rice-Evans method [13]. The absorbance was measured in a SEMCO spectrophotometer at 535 $\mathrm{nm}$ in $1-\mathrm{cm}$ cuvettes. The TBARS expressed in micromoles per liter were calculated based on the absorbance value, using the molar extinction coefficient for TBARS $\left(\varepsilon=1.56 \times 10^{5} \mathrm{M}^{-1} \times \mathrm{cm}^{-1}\right)$. All estimations were performed twice, including control plasma samples, in which spontaneous lipid peroxidation, without the influence of the drug, was measured.

\section{Statistical Analysis}

The results were subjected to statistical analysis (mean values and standard error of the mean). The significance of differences in TBARS levels (drug-treated samples vs. control) was calculated using the paired Student's t test. The two-way ANOVA test was used. Post hoc comparisons for the TBARS levels were carried out with the NIR test. Statistica v. 6.0 by Statsoft, Inc. was used.

\section{Results}

The comparative study in vitro has shown that after incubation of plasma with tested antipsychotics, plasma lipid peroxidation (measured as TBARS level) was changed. The analysis of TBARS levels after the incubation of plasma with the drugs (QUE, OLA and HAL) showed significant differences in comparison to the control values. The two-way ANOVA test showed that the differences in TBARS levels significantly depended on the studied drug (drugs at lower concentrations: $p=5.3$ $\times 10^{-4}$, and at higher concentrations: $\left.\mathrm{p}=2.2 \times 10^{-5}\right)$, but did not depend on the time of incubation $(\mathrm{p}>0.05)$ and interaction $(p>0.05)$.

In the post hoc analysis (NIR test), some significant differences in the TBARS levels between QUE and HAL were found (fig. 1). After $24 \mathrm{~h}$ of incubation of plasma with QUE or HAL (at lower and higher concentrations), the differences in TBARS levels between drugs $(\mathrm{p}=5.9 \times$ $10^{-4}, \mathrm{p}=2.2 \times 10^{-5}$, respectively) were significant as well as after $1 \mathrm{~h}$ of incubation ( $\mathrm{p}=0.04, \mathrm{p}=0.02$, respectively). OLA (1-hour incubation), contrary to HAL and QUE, did not cause significant changes in the level of plasma TBARS. After 24-hour incubation of plasma with OLA (at higher concentrations), an increased level of TBARS was observed $(p=0.01)$ (fig. 1). 
Fig. 1. The comparison of QUE, OLA and HAL effects on plasma lipid peroxidation (in vitro; expressed as TBARS levels). Data were calculated as differences in comparison with data of controls (without drugs). The asterisk indicates significance (NIR test). Drug concentrations: HAL, 4 and 20 ng/ml; QUE, 175 and 275 ng/ml; OLA, 20 and $40 \mathrm{ng} / \mathrm{ml}$.

Fig. 2. The effects of drugs (incubation $24 \mathrm{~h}$ ) on lipid peroxidation of human plasma expressed as TBARS level. HAL: 4 and $20 \mathrm{ng} / \mathrm{ml}$; QUE: 175 and $275 \mathrm{ng} / \mathrm{ml}$; OLA: 20 and $40 \mathrm{ng} / \mathrm{ml}$; control samples (without drugs).
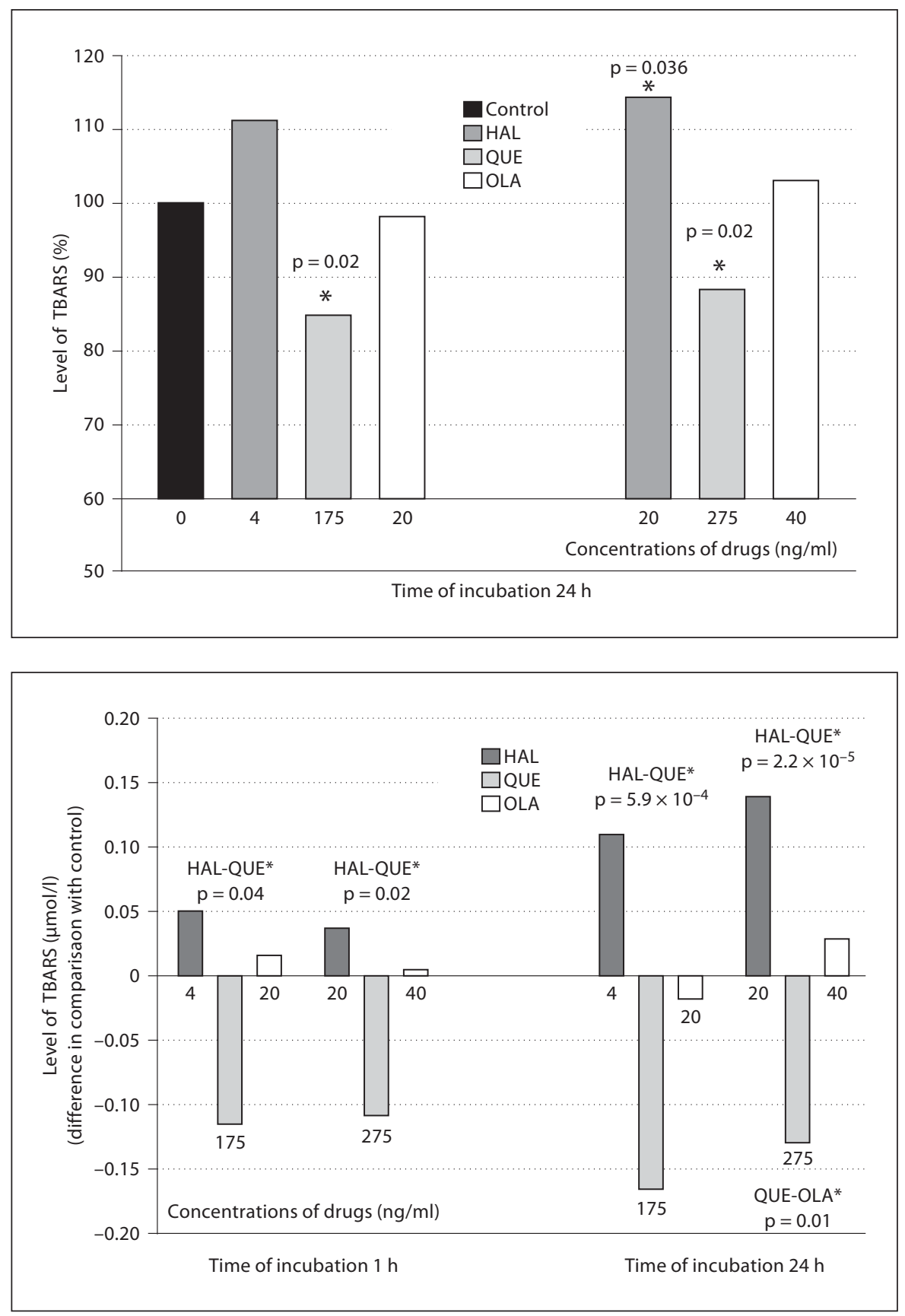

QUE after 1 and $24 \mathrm{~h}$ of incubation caused a significant decrease (about 8 and 15\%, respectively) in plasma lipid peroxidation (expressed as TBARS) in comparison with control $(p<0.02)$ (fig. 2). OLA caused no changes of TBARS levels $(p>0.05)$. Contrary to QUE and OLA, HAL, especially at the higher concentration $(20 \mathrm{ng} / \mathrm{ml})$ and after $24 \mathrm{~h}$, caused a significant increase (about 14\%, $\mathrm{p}=0.036$ ) in TBARS in plasma (fig. 2).

\section{Discussion}

Oxidative stress is a state when there is an imbalance between ROS generation and antioxidant defenses in favor of the former. There are multiple pathological consequences of increased ROS production. ROS induce oxidative stress and damage to all types of biomolecules including lipids and are involved in various pathophysi-

Neuropsychobiology 2011;63:197-201 
ological processes and disorders. Oxidative stress and the oxidative changes in different biomolecules may be involved in the pathology of schizophrenia [14]. The abnormalities and peroxidation of membrane phospholipids and polyunsaturated fatty acids caused by oxidative stress play an important role in etiopathogenic mechanisms of schizophrenia [15-21]. The changes of membrane dysfunction caused by lipid peroxidation seem to be secondary to ROS generation and may contribute to specific aspects of schizophrenic symptomatology and complications of its treatment $[22,23]$. Our earlier results have shown that the level of TBARS was significantly increased in plasma of patients with schizophrenia [21,24], whereas the activities of antioxidant defense enzymes were diminished [24]. We have also found oxidative stress, measured as isoprostane level (8-iso- $\left.\mathrm{PGF}_{2 \alpha}\right)$, in the urine of schizophrenia patients [24]. The increase in lipid peroxidation products in plasma of first-episode patients with schizophrenia (no antipsychotic drugs) has also been described $[20,25]$. Therapy with antipsychotics may affect lipid metabolism. Oxidative injury in schizophrenia can be caused by pathophysiological processes of the disease and probably also by treatment with antipsychotics [2628]. An increase in lipid peroxidation was observed in platelets [21] and in plasma [24, 28, 29] measured by TBARS level during treatment with antipsychotics. Dietrich-Muszalska [28] demonstrated an increased plasma lipid peroxidation after incubation of human plasma of healthy subjects with HAL (in vitro). Pai et al. [30] and Lohr et al. [2] in the 1990s found that oxidative stress contributed to the toxicity of HAL, which activated a sequence of cellular processes leading to cell death and the production of ROS that was an integral part of that cascade. An experimental study on animals showed an elevated level of lipid peroxidation and peroxidative neuronal injury caused by HAL [31]. The toxicity of HAL leads to changes in mitochondrial membrane potential, generation of ROS, decrease in the concentration of glutathione and increase in intracellular $\mathrm{Ca}^{2+}$ both in cortical and hippocampal neurons [31]. Increased lipid peroxidation in cerebrospinal fluid and plasma of TD patients was observed, and antipsychotics-mediated oxidative injury was suggested in the development of TD [2, 5, 29, 32]. The risk of appearance of EPS during the treatment with SGAs is significantly smaller, especially in patients treated with QUE and OLA, and depends on the doses of these drugs $[8,10,33]$. SGAs such as QUE and OLA at lower doses might not significantly influence the increase in oxidative stress, measured by the level of lipid peroxidation. Kropp et al. [34] showed that the concentration of malondialdehyde after 3 weeks of pharmacotherapy was significantly higher in the group treated with HAL than in that treated with SGAs (clozapine, risperidone, QUE, and amisulpride). Dakhale et al. [35] found a tendency to a decrease in the lipid peroxidation level after treatment with SGAs. Comparative studies showed that SGAs caused significantly less oxidative damage than HAL [35, 36]. Our study indicates that HAL action was significantly different from QUE and OLA, since it produced a significantly higher level of TBARS. The effects of HAL observed in vitro may occur in vivo, i.e. in HAL-treated patients. Schizophrenic patients are usually treated with antipsychotics for a very long time and pharmacodynamic properties during long-term treatment are unknown. The present study showed a significant difference between actions of these drugs (in vitro). QUE at higher doses has a significant antioxidant effect. Similar data were demonstrated by $\mathrm{Xu}$ et al. [37]. They showed that QUE protects cultured cells against oxidative stress-related cytotoxicities induced by amyloid- $\beta$ by blocking hydroxyl radical generation induced by amyloid. QUE by eliminating hydroxyl radical attenuates oxidative stress thus protecting brain cells against oxidative-stress-related damage and improving cognitive function in patients with schizophrenia [37]. The antioxidant mechanism of QUE may contribute to the low incidence of EPS and movement disorders caused by this drug in patients with schizophrenia, and may be effective in treating TD caused by other antipsychotics [38].

So far, we can compare our data to the results of 1 clinical study; only Kropp et al. [34] demonstrated in vivo antioxidant effect of QUE. The results of our study in vitro show that first-generation antipsychotics and SGAs at doses recommended for the acute episode of schizophrenia treatment have different effects on plasma lipid peroxidation. QUE, contrary to HAL, possesses antioxidant properties. The mechanism of pro- and antioxidant action of tested antipsychotics on lipids in plasma is not known and requires further studies.

\section{Acknowledgement}

This study was supported by grant 502-11-176 from Medical University of Lodz, Poland. 


\section{References}

1 Margolese HC, Chouinard G, Kolivakis TT, Beauclair L, Miller R: Tardive dyskinesia in the era of typical and atypical antipsychotics. 1. Pathophysiology and mechanisms of induction. Can J Psychiatry 2005;50:541-547.

-2 Lohr JB, Kuczenski R, Bracha HS, Moir M, Jeste DV: Increased indices of free radical activity in the cerebrospinal fluid of patients with tardive dyskinesia. Biol Psychiatry 1990;15:535-539.

3 Kulkarni SK, Naidu PS: Tardive dyskinesia: an up date. Drugs Today 2001;137:97-119.

$\checkmark 4$ Balijepalli S, Kenchappa RS, Boyd MR, Ravindranath $\mathrm{V}$ : Protein thiol oxidation by haloperidol results in inhibition of mitochondrial complex I in brain regions: comparison with atypical antipsychotics. Neurochem Int 2001;38:425-435.

$\checkmark 5$ Cadet JL, Lohr JB, Jeste DV: Free radicals and tardive dyskinesia. Trends Neurosci 1986;9: 107-108.

6 Sachdev PS: The current status of tardive dyskinesia. Aust NZ J Psychiatry 2000;34: 355-369.

7 Cadet JL, Lohr JB, Jeste DV: Tardive dyskinesia and schizophrenic burnout: the possible involvement of cytotoxic free radicals; in Henn FA, DeLisi LE (eds): Handbook of Schizophrenia: The Neurochemistry and Pharmacology of Schizophrenia. Amsterdam, Elsevier, 1987, pp 425-438.

$\checkmark 8$ Möller HJ: Novel antipsychotics in the long term treatment of schizophrenia. World J Biol Psychiatry 2004;5:9-19.

9 Hong WW, Rak IW, Ciuryla VT, Wilson AM, Kylstra JW, Meltzer HY, Carpenter WTJR, Lehman A, Arvanitis LA: Medicalclaims databases in the design of a healthoutcomes comparison of quetiapine ('Seroquel') and usual-care antipsychotic medication. Schizophr Res 1998;22:51-58.

-10 Falkai P, Wobrock T, Lieberman J, Glenthoj B, Gattaz WF, Möller HJ, WFSBP Task Force on Treatment Guidelines for Schizophrenia: World Federation of Societies of Biological Psychiatry (WFSBP) guidelines for biological treatment of schizophrenia. 1. Acute treatment of schizophrenia. World J Biol Psychiatry 2005;6:132-191.

-11 Sacchetti E, Valsecchi P: Quetiapine, clozapine, and olanzapine in the treatment of tardive dyskinesia induced by first-generation antipsychotics: a 124-week case report. Int Clin Psychopharmacol 2003;18:357-359.

-12 Sheehan DV, Lecrubier Y, Sheehan KH, Amorim P, Janavs J, Weiller E Hergueta T, Baker R, Dunbar GC: The Mini-International Neuropsychiatric Interview (M.I.N.I.): the development and validation of a structured diagnostic psychiatric interview for DSM-IV and ICD-10. J. Clin Psychiatry 1998;59:2233.

13 Rice-Evans CA: Formation of free radicals and mechanisms of action in normal biochemical processes and pathological states; in Rice-Evans CA, Burdone RH (eds): Free Radical Damage and Its Control. Amsterdam, Elsevier, 1994, pp 131-153.

14 Berk M, Copolov D, Dean O, Lu K, Jeavons S, Schapkaitz I, Anderson-Hunt M, Judd F, Katz P, Ording-Jespersen S, Little J, Conus P, Cuenod M, Do KQ, Bush AI: N-acetyl cysteine as a glutathione precursor for schizophrenia - A double-blind, randomized, placebo-controlled trial. Biol Psychiatry 2008; 64:361-368.

15 Horrobin DF: The membrane phospholipids hypothesis as a biochemical basis for the neurodevelopmental concept of schizophrenia. Schizophr Res 1998;30:193-208.

16 Fenton WS, Hibbeln J, Knable M: Essential fatty acids, lipid membrane abnormalities and the diagnosis and treatment of schizophrenia. Biol Psychiatry 2000;47:8-21.

17 Ross BM: Phospholipid and eicosanoid signaling disturbances in schizophrenia. Prostaglandins Leukot Essent Fatty Acids 2003; 69:407-412.

18 Mahadik SP, Scheffer RE: Oxidative injury and potential use of antioxidants in schizophrenia. Prostaglandins Leukot Essent Fatty Acids 1996;55:45-54.

19 Fendri C, Mechri A, Khiari G, Othman A Kerkeni A, Gaha L: Oxidative stress involvement in schizophrenia pathophysiology. Encephale 2006;32:244-252.

20 Arvindakshan M, Sitasawad S, Debsikdar V, Ghate M, Evans D, Horrobin DF, Bennett C, Ranjekar PK, Mahadik SP: Essential polyunsaturated fatty acid and lipid peroxide levels in never-medicated and medicated schizophrenia patients. Biol Psychiatry 2003;53:56-64.

21 Dietrich-Muszalska A, Olas B, Rabe-Jablonska J: Oxidative stress in blood platelets from schizophrenic patients. Platelets 2005;16: 386-391.

22 Du Bois TM, Deng C, Huang XF: Membrane phospholipid composition, alterations in neurotransmitter systems and schizophrenia. Prog Neuropsychopharmacol Biol Psychiatry 2005;29:878-888.

23 Zhang XY, Tan YL, Cao LY, Wu GY, Xu Q Shen Y, Zhou DF: Antioxidant enzymes and lipid peroxidation in different forms of schizophrenia treated with typical and atypical antipsychotics. Schizophr Res 2006;81: 291-300.

24 Dietrich-Muszalska A, Olas B: Isoprostanes as indicators of oxidative stress in schizophrenia. World J Biol Psychiatry 2007;4:1-6.

25 Mahadik SP, Mukherjee S, Correnti EE, Scheffer R: Elevated levels of lipid peroxidation products in plasma of drug-naive patients at the onset of psychosis. Schizophr Res 1995;15:66.

26 Peet M, Laugharne J, Rangarajan N, Reynolds GP: Tardive dyskinesia, lipid peroxidation, and sustained amelioration with vitamin E treatment. Int Clin Psychophamacol 1993;8:151-153.
27 Gama CS, Salvador M, Andreazza AC, Kapczinski F, Silva Belmonte-de-Abreu P: Elevated serum superoxide dismutase and thiobarbituric acid reactive substances in schizophrenia: a study of patients treated with haloperidol or clozapine. Prog Neuropsychopharmacol Biol Psychiatry 2006;30: 512-515.

28 Dietrich-Muszalska A: The impact of haloperidol on lipids peroxidation in human blood platelets and plasma at in vitro studies. Psychiatr Psychol Klin 2004;3:150-156.

29 Gama CS, Salvador M, Andreazza AC, Lobato MI, Berk M, Kapiczinski F, Belmontede-Abreu PS: Elevated serum thiobarbituric acid reactive substances in clinically symptomatic schizophrenic males. Neurosci Lett 2008;433:270-273.

$\checkmark 30$ Pai BN, Janakiramaiah N, Gangadhar BN, Ravindranath V: Depletion of glutathione and enhanced lipid peroxidation in the CSF of acute psychotics following haloperidol administration. Biol Psychiatry 1994;36:489491.

31 Sagara Y: Induction of reactive oxygen species in neurones by haloperidol. J Neurochem 1998;71:1002-1012.

32 Zhang XY, Tan YL, Zhou DF, Cao LY, Wu GY, Haile CN, Kosten TA, Kosten TR: Disrupted antioxidant enzyme activity and elevated lipid peroxidation products in schizophrenic patients with tardive dyskinesia. J Clin Psychiatry 2007;68:754-760.

33 Miyamoto S, Duncan GE, Marx CE, Lieberman JA: Treatments for schizophrenia: a critical review of pharmacology and mechanisms of action of antipsychotic drugs. Mol Psychiatry 2005;10:79-104.

-34 Kropp S, Kern V, Lange K, Degner D, Hajak G, Kornhuber J, Ruther E, Emrich HM, Schneider U, Bleich S: Oxidative stress during treatment with first- and second-generation antipsychotics. J Neuropsychiatry Clin Neurosci 2005;17:227-231.

-35 Dakhale G, Khanzode S, Khanzode S, Saoji A, Khobragade L, Turankar A: Oxidative damage and schizophrenia: the potential benefit by atypical antipsychotics. Neuropsychobiology 2004;49:205-209.

36 Parikh V, Khan MM, Mahadik SP: Differential effects of antipsychotics on expression of antioxidant enzymes and membrane lipid peroxidation in rat brain. J Psychiatr Res 2003;37:43-51.

>37 Xu H, Wang H, Zhuang L, Yan B, Yingxin Y, Wei Z, Zhang Y, Dyck LE, Richardson SJ, He J, Li X, Kong J, Li XM: Demonstration of an anti-oxidative stress mechanism of quetiapine. FEBS J 2008;275:3718-3728.

-38 Vesely C, Kufferle B, Brucke T, Kacper S: Remission of severe tardive dyskinesia in a schizophrenic patient treated with the atypical antipsychotic substance quetiapine. Int Clin Psychopharmacol 2000;15:57-60. 